Medical Research Archives. Volume 5, issue 7. July 2017.

\title{
Cystatins as regulators of cancer
}

\author{
Authors \\ James L. Cox \\ AT Still University, \\ Department of \\ Biochemistry, Kirksville, \\ Missouri, USA \\ jcox@atsu.edu
}

\begin{abstract}
The cystatins, natural inhibitors of cysteine proteases, act as metastasis suppressors for a wide variety of different cancers. An update of the various actions of cystatins in cancer is the focus of the current review. In the progression of cancer cells, besides certain genetic changes, augmentation of invasion occurs with an increase in cysteine protease (cathepsin) activity. Because the cystatins are key regulators of the cathepsins, they too play critical roles in cancer. All aspects of metastasis are influenced by the cystatins in a variety of cancers. For some cancers cystatins can be used as prognostic factors. New roles of the cystatins in cancer as gene regulatory proteins are being advanced. Overexpression of cystatins has been found to inhibit metastasis and angiogenesis in certain cancers. The cystatins may introduce future therapeutic advances in the control of cancer.
\end{abstract}

Keywords: cystatin, cathepsin, stefin, cancer, metastasis, cysteine protease inhibitor 
Medical Research Archives. Volume 5, issue 7. July 2017.

\section{Cystatins as regulators of cancer}

\section{Introduction}

The progression of normal cells to invasive, often metastatic cancer cells involves overexpression of cysteine proteases or cathepsins. Other protease families show increased expression in cancer cells, but the cathepsins have multiple roles which relate to the aggressiveness of most cancers. While cathepsins are normally lysosomal, cancer cells often overexpress these enzymes on the cell surface and/or secrete them (Klose et al., 2006). The cancer cell associated cathepsins are involved in many cancer cell functions including extracellular matrix (ECM) degradation, invasion, proliferation, and angiogenesis (Gocheva et al., 2006). Key regulators of the various cancer cell associated cathepsins are the cystatin family of cysteine proteases inhibitors. New therapeutic modalities which target tumor associated cathepsin activities are greatly anticipated to rein in tumor growth and metastasis. The complexities of targeting tumor cell associated cathepsins with small molecular weight inhibitors has been touched on elsewhere so here the focus will be mainly on inhibition of cancer by the cystatins (Olson and Joyce, 2015) .

\section{Cathepsins in cancer}

Multiple cysteine protease such as cathepsins $\mathrm{B}, \mathrm{C}, \mathrm{H}, \mathrm{K}, \mathrm{L}, \mathrm{S}$, and $\mathrm{Z}$ are found primarily in lysosomes in normal cells. Malignant tumors display an upregulation of cathepsin B. Knockout of cathepsin B in a mouse tumor model increased tumor cell death, lowered cell proliferation, decreased cell invasion, and reduced tumor vascularization (Gocheva et al., 2006). One target for cathepsin B cleavage was found to be E-cadherin, the level of which is pertinent to tumor cell invasion. Multiple extracellular matrix proteins can also be degraded by cathepsin $\mathrm{B}$ during tumor cell invasion. It is thought cathepsin B can also degrade extracellular inhibitors of angiogenesis and hence stimulate this process in the tumor vicinity. One problem with targeting solely cathepsin B as an anticancer strategy however is the compensation by other cathepsins (Gocheva and Joyce, 2007).

Cathepsin $\mathrm{L}$ expression in cancers often parallels cathepsin B overexpression. CLIK-148, a selective cathepsin L inhibitor, was able to partially block invasion in glioblastoma cells (Zajc et al., 2006). Work on monotherapeutic cysteine protease inhibitors has led to the general conclusion that broader inhibitors will be more successful against tumor cell invasion. Combination therapy of a cathepsin L inhibitor plus chemotherapy showed increased effectiveness by reducing cancer drug resistance (Bell-McGuinn et al., 2007).

Tumor and tumor associated cells contribute cathepsin $\mathrm{S}$ for tumor growth and angiogenesis (Small et al., 2013). Loss of cathepsin $\mathrm{S}$ expression decreased tumor angiogenesis and reduced proliferation while increasing apoptosis. In breast cancer cathepsin S may also contribute to chemotherapeutic resistance (Shree et al., 2011). A class of ketoamides is being tested as cathepsin $\mathrm{S}$ inhibitors for several cancer types (Chen et al., 2010). While there are other cathepsins that have been associated 


\section{Cystatins as regulators of cancer}

with cancer cells, they have been less well studied than the several mentioned above.

\section{Cystatins}

The cystatins are a superfamily of cysteine protease inhibitors that are produced by virtually all eukaryotic cells (Turk et al., 2008). Three basic types of cystatins are the type I stefins, the type II cystatins, and the type III cystatins or kininogens. The stefins, chiefly stefins A and B, are found primarily intracellularly and are 100 amino acids in length. The type II cystatins are slightly longer than the stefins at around 120 amino acids with typically two intrachain disulfide bonds and are mainly secreted proteins. The type three cystatins, kininogens, are more complex with three cystatin motifs, and also a terminal kinin peptide sequence. As kininogens are often found at low levels chiefly in serum they will not enter into further discussion in this review. For further information on this topic see the papers by Kashuba (Kashuba et al., 2013a; Kashuba et al., 2013b).

The role of cystatins in cancer has been expanding over the past several years. The chief role of cystatins is the control of excessive cathepsin activity that could be released from lysosomes or from dead or dying cells. As for cancer, the cystatins are probably not defensive proteins against tumor development, but rather, happen to have functions that pertain to metastasis suppression. As a general rule cystatin expression is decreased in many but certainly not all cancers (Rivenbark and Coleman, 2009). More commonly in cancer it might be appropriate to say the ratio of cathepsin to cystatin is increased. It is certainly true that the cystatins have roles in metastasis suppression that extend beyond action as cysteine protease inhibitors. This short review will cover new findings on cystatins and cancer since our 2009 paper on this topic (Cox, 2009). In addition, several pathological conditions such as cardiovascular disease, neurodegeneration, and arthritis are associated with excessive cathepsin levels which may overwhelm cystatin levels and contribute to tissue degradation (Weiss-Sadan et al., 2017).

\section{Type I cystatins}

Stefin A expression is inversely associated with lymph node metastasis, recurrence and survival in laryngeal cancer (Li et al., 2011). Upregulation of stefin A significantly inhibits invasion, migration, and proliferation for laryngeal cancer cells. It was also suggested stefin A might be used as a diagnostic marker for this cancer. The increased expression of stefins $\mathrm{A}$ and $\mathrm{B}$ are however found in hepatocellular carcinoma (Lin et al., 2016). Both protein and mRNA levels were increased in cancerous tissues versus surrounding normal tissue. In fact, the levels of cathepsins B and D were also significantly elevated suggesting a critical role of this enzyme-inhibitor ratio in the progression of hepatocellular carcinoma.

In a study of immunohistochemistry for head and neck cancers the levels of cathepsins and stefins $\mathrm{A}$ and $\mathrm{B}$ were examined (Anicin et al., 2013). Stefin A immunopositivity correlates with a favorable outcome in this study of head and neck cancer. In a related study by this group a three-fold disease recurrence was found for 


\section{Cystatins as regulators of cancer}

stefin A downregulation compared to upregulation (Strojan et al., 2011).

The role of stefin B has been examined in breast cancer with Stfb null mice (Butinar et al., 2014). In transgenic PyMT mice that were stefin B knockout no effect on proliferation, angiogenesis, migration, and spontaneous cell death was noted. No effect on metastasis was seen in knockout mice, however, reduced size of mammary tumors was detected. Tumor cells from these mice were highly sensitive to induction of apoptosis with oxidative stress. Stefin B may be a protective factor against apoptosis, particularly induction of cell death through the lysosomal pathway. Leakage of cathepsins from lysosomes is known to induce apoptosis by cleavage of $\mathrm{Bid}$ and other anti-apoptotic proteins (DrogaMazovec et al., 2008). Stefin B and other cytoplasmic cysteine protease inhibitors can block the apoptotic actions of the cathepsins. More work is required to elucidate the role of stefin $\mathrm{B}$ in cancer progression, particularly its role in protection of the cell against apoptosis.

\section{Type II cystatins}

Both cystatin C and cathepsin B protein and mRNA levels were elevated in esophageal cancer patient samples (Yan et al., 2015). It is speculated the high cystatin $\mathrm{C}$ levels might be due to induction by cathepsin B. Overexpression of cystatin C in esophageal carcinoma cells dramatically reduces cell invasion but does not influence cell proliferation. Reduction of cystatin $\mathrm{C}$ by RNAi dramatically increases invasion in this cell system. Neither up nor down regulation of cystatin significantly alters levels of apoptosis of these cancer cells in culture. In another cell system, in response to oxidative stress, overexpression of cystatin C protects $\mathrm{PC} 12$ cells against apoptosis (Nishiyama et al., 2005). It is believed oxidative damage to lysosomal membranes causes leakage of cathepsins as a trigger for apoptosis in this system. In prostate cancer patients, lower levels of cystatin C correlates with lower survivial (Wegiel et al., 2009). Targeting cystatin C with RNAi leads to increased invasiveness of prostate cancer cells. Conversely, overexpression of cystatin $\mathrm{C}$ reduces invasion of PC 3 cells. Some evidence of cystatin $\mathrm{C}$ inhibition of MAPK/Erk signaling was found which may be tied to decreased invasion in prostate cancer cells.

Cystatin D has a more restricted tissue distribution, being found primarily in submandibular and parotid glands. Research has shown cystatin D has a tumor suppressor effect also in colorectal cancer (Alvarez-Diaz et al., 2009). Cystatin D was found to be inducible with vitamin $\mathrm{D}$ in mice and responsible for the major tumor suppressor action of vitamin D in colorectal cancer. Poorly differentiated cancer cells in general have lower levels of cystatin D expression. The decreased proliferation of colon cancer cells with cystatin D overexpression is linked to c-MYC suppression in this system. In some manner, cystatin D expression interferes with EMT (epithelial mesenchymal transition) through decreases in Snail expression and increases E-cadherin levels leading to restriction of migration of cancer cells. Interestingly, Hunten and Hermeking also show cystatin D is induced upon tumor suppressor p53 
Medical Research Archives. Volume 5, issue 7. July 2017.

\section{Cystatins as regulators of cancer}

activation in colorectal tumor cells (Hunten and Hermeking, 2015).

Cystatin D has been localized to nuclear DNA and is found to be involved in the transcription of multiple genes in human colon cancer cells (Ferrer-Mayorga et al., 2015). Several genes related to the tumor suppressor role of cystatin D are the cytokines CX3CL1 and oncostatin M as well as FGF-4 and RUNX 1 and 2 transcription factors. The action of cystatin $\mathrm{D}$ in the nucleus appears to be independent of protease inhibition. Knockdown of cystatin D levels confirms gene regulation functions for this molecule. Future studies are required for a more mechanistic look at cystatin D in gene transcription as well as extension to other cancer types. Cystatin $\mathrm{F}$ is expressed primarily in immune cells and its target for inhibition is cathepsin C. Very little is known about the role of cystatin $\mathrm{F}$ in cancer at present.

Cystatin $\mathrm{M} / \mathrm{E}$ (mammary/epithelial) is downregulated in metastatic breast cancer in comparison to primary tumors (Sotiropoulou et al., 1997). Cystatin M/E exogenous expression in metastatic breast cancer cells decreases proliferation, invasion, migration and endothelial cell adhesion (Shridhar et al., 2004). Zhang et al. found cystatin M/E reduces primary tumor growth but not metastatic seeding of lung tissues (Zhang et al., 2004). Epigenetic silencing of cystatin $\mathrm{M} / \mathrm{E}$ by promoter methylation has been found for glioma and breast cancer cells (Rivenbark and Coleman, 2009) (Qiu et al., 2008). Expression of cystatin $\mathrm{M} / \mathrm{E}$ is also downregulated in metastatic prostate cancer cell lines (Pulukuri et al., 2009). Induction of cystatin $\mathrm{M} / \mathrm{E}$ occurs upon treatment of prostate cancer cell lines with tricostatin, a histone deacetylase inhibitor. Overexpression of cystatin $\mathrm{M} / \mathrm{E}$ in prostate cancer cell lines reduces invasion and cell proliferation. Overexpression of cystatin $\mathrm{M}$ in MDA-MB-435 cells also led to a decrease in proliferation of the cells (Shridhar et al., 2004). Interestingly a broad spectrum cysteine protease inhibitor is not able to inhibit cell proliferation, while it was able to partially inhibit tumor cell invasion. In a separate study, cystatin $\mathrm{M}$ was found to inhibit colony formation, proliferation, migration and invasion of breast cancer cells (Jin et al., 2012). Proteomic analysis of metastatic breast cancer cell lines reveals cystatin $\mathrm{M}$ to be a key downregulated factor in bone metastasis for these cells. Overexpression of cystatin $\mathrm{M}$ decreases osteolytic metastasis in mice of breast cancer cells. Since cathepsin K is implicated in osteolytic bone degradation it will be of great interest to look at cathepsin $\mathrm{K}$ involvement in bone metastasis.

Cystatin SN (salivary, neutral) is found to be upregulated in gastric cancers compared to noncancerous tissues and correlated with a tumor staging system (Choi et al., 2009). Downregulation of cystatin SN with siRNA both decreases cell proliferation and increases cathepsin activity in gastric cancer cells. In contrast, cystatin SN levels decrease in esophageal cancer cells relative to non-cancerous tissues from patients (Chen et al., 2013). Survival of esophageal cancer patients was significantly greater with high cystatin SN levels and the authors suggest levels might be used as a prognostic indicator for this cancer type. The difference 
Medical Research Archives. Volume 5, issue 7. July 2017.

\section{Cystatins as regulators of cancer}

in cystatin $\mathrm{SN}$ expression between the different cancer types might be due to stage differences or tissue type, squamous cell carcinoma versus adenocarcinoma.

We have expressed several cystatin $\mathrm{C}$ variants in B16 F10 melanoma cells to determine the critical region of cystatin for melanoma metastasis inhibition (McIntire, 2015). Basically, mutation of four amino acids in the first hairpin turn of cystatin $\mathrm{C}$ eliminates migration and invasion inhibition by cystatin. An N-terminal deletion of 10 amino acids for cystatin, which should decrease cysteine protease inhibitor activity, fails to decrease melanoma cell invasion inhibition. We are unsure of the cellular target for the active region of cystatin but are looking into several signaling pathways at the present. Our hunch is that cystatins may be behaving differently in different tumor types. In tumor types where cystatins are overexpressed, cystatin may be playing a protective role and these cancer cells use signaling pathways which are not inhibited by cystatin. Alternatively, in tumor types where cystatin expression is decreased and behaving like a metastasis suppressor, other signaling pathways are engaged which are potentially inhibited by cystatin.

Recombinant snake venom cystatin has been shown to inhibit metastasis of B16F10 melanoma when administered intravenously and spontaneous metastasis when administered intraperitoneally to mice (Xie et al., 2013a). In other work with liver cancer cells, expression of snake venom cystatin inhibits invasion and metastasis in vitro and in vivo (Tang et al., 2011). The authors attribute some of the anti-invasion effects to changes in EMT indicated by changes in E-cadherin and Twist expression with cystatin overexpression. A decrease in cathepsin B activity was also noted in liver cancer cells with cystatin overexpression, so protease inhibition may also be involved in this system. Surprisingly, snake venom cystatin also effectively inhibits angiogenesis, again with the B16F10 tumor model in mice (Xie et al., 2013b). Recombinant cystatin was able to inhibit capillary tube formation with as little as 10 $\mathrm{ug} / \mathrm{ml}$ cystatin. Cystatin was able to suppress secretion of VEGF and bFGF, both potent inducers of angiogenesis, although the mechanism needs further study.

A cystatin-like protein derived from myeloid suppressor cells has been found to inhibit metastasis of mammary 4T1 cells (Boutte et al., 2011). This cystatin, termed NGP (neutrophilic granule protein), not only inhibits metastasis, but also inhibits angiogenesis and increases tumor cell necrosis and apoptosis. A novel finding was conditioned media from $4 \mathrm{~T} 1$ cells was able to suppress expression of the cystatin in the myeloid cells. This finding might suggest other cancers also produce secreted suppressor molecules for other cystatins to in effect "suppress the suppressor cystatin activity" in the local tumor microenvironment. Hopefully more details will emerge on this exciting possibility.

\section{Future questions}

Although a body of knowledge is coming together on cystatin roles in cancer, a number of questions remain. The level of free cystatin in and around cancer cells would be nice to know such that when 
Medical Research Archives. Volume 5, issue 7. July 2017.

\section{Cystatins as regulators of cancer}

bound to cathepsin, loss of cystatin activity is presumed. We do not even know how much of the anti-cancer effects of cystatins are extracellular versus intracellular. Careful analysis of gene expression changes due to cystatin role in transcription is critical to understanding how these changes affect tumor cell invasion and metastasis. New insights into cathepsin involvement in cancer progression are also needed for mechanistic understanding of cancer invasion. The roles of cystatins in such processes as tumor angiogenesis and tumor immunity also need to be addressed in future studies.

\section{Summary}

Since cysteine proteases play such critical roles that foster tumor invasion and metastasis, the cystatins will assume greater importance in efforts to control cancer invasiveness. Much more needs to be learned about how cystatins expression levels are regulated, both in health and in diseases such as cancer. Delivery of cystatins to patients with metastatic disease may be a future therapy to arrest cancer spread and to curtail tumor growth. Certain cancers may be inhibited in their spread by cysteine protease inhibition by the cystatins. Others may be blocked by non-cysteine protease inhibitor functions of the cystatins such as signal transduction inhibition of cancer cells or regulation of cancer suppressor genes. Many hurdles will have to be overcome to efficiently deliver cystatins systemically to distantly spread tumors. Advances in nanotechnology and other methods of delivery of cystatins selectively to cancer cells will be welcome technological partners. Other metastasis suppressors, which share common pathways with the cystatins, may also come to the forefront in future cancer therapies. The cystatins, due to their anti-metastatic actions on a wide range of different cancers, will figure prominently as metastasis suppressors for new types of attacks on metastatic disease. Coupling cystatin suppression of metastasis with other simultaneous treatment modalities may have much better chances of long-term success for cancer treatment. 


\section{Cystatins as regulators of cancer}

\section{References}

Alvarez-Diaz, S., N. Valle, J.M. Garcia, C. Pena, J.M. Freije, V. Quesada, A. Astudillo, F. Bonilla, C. Lopez-Otin, and A. Munoz. 2009. Cystatin D is a candidate tumor suppressor gene induced by vitamin $\mathrm{D}$ in human colon cancer cells. J Clin Invest. 119:2343-2358.

Anicin, A., N. Gale, L. Smid, J. Kos, and P. Strojan. 2013. Expression of stefin A is of prognostic significance in squamous cell carcinoma of the head and neck. Eur Arch Otorhinolaryngol. 270:3143-3151.

Bell-McGuinn, K.M., A.L. Garfall, M. Bogyo, D. Hanahan, and J.A. Joyce. 2007. Inhibition of cysteine cathepsin protease activity enhances chemotherapy regimens by decreasing tumor growth and invasiveness in a mouse model of multistage cancer. Cancer Res. 67:7378-7385.

Boutte, A.M., D.B. Friedman, M. Bogyo, Y. Min, L. Yang, and P.C. Lin. 2011. Identification of a myeloid-derived suppressor cell cystatin-like protein that inhibits metastasis. FASEB J. 25:2626-2637.

Butinar, M., M.T. Prebanda, J. Rajkovic, B. Jeric, V. Stoka, C. Peters, T. Reinheckel, A. Kruger, V. Turk, B. Turk, and O. Vasiljeva. 2014. Stefin B deficiency reduces tumor growth via sensitization of tumor cells to oxidative stress in a breast cancer model. Oncogene. 33:3392-3400.
Chen, J.C., B.J. Uang, P.C. Lyu, J.Y. Chang, K.J. Liu, C.C. Kuo, H.P. Hsieh, H.C. Wang, C.S. Cheng, Y.H. Chang, M.D. Chang, W.S. Chang, and C.C. Lin. 2010. Design and synthesis of alpha-ketoamides as cathepsin $\mathrm{S}$ inhibitors with potential applications against tumor invasion and angiogenesis. J Med Chem. 53:45454549.

Chen, Y.F., G. Ma, X. Cao, R.Z. Luo, L.R. He, J.H. He, Z.L. Huang, M.S. Zeng, and Z.S. Wen. 2013. Overexpression of cystatin SN positively affects survival of patients with surgically resected esophageal squamous cell carcinoma. BMC Surg. 13:15.

Choi, E.H., J.T. Kim, J.H. Kim, S.Y. Kim, E.Y. Song, J.W. Kim, S.Y. Kim, Y.I. Yeom, I.H. Kim, and H.G. Lee. 2009. Upregulation of the cysteine protease inhibitor, cystatin SN, contributes to cell proliferation and cathepsin inhibition in gastric cancer. Clin Chim Acta. 406:45-51.

Cox, J.L. 2009. Cystatins and cancer. Front Biosci (Landmark Ed). 14:463-474.

Droga-Mazovec, G., L. Bojic, A. Petelin, S. Ivanova, R. Romih, U. Repnik, G.S. Salvesen, V. Stoka, V. Turk, and B. Turk. 2008. Cysteine cathepsins trigger caspase-dependent cell death through cleavage of bid and antiapoptotic Bcl-2 homologues. $J$ Biol Chem. 283:19140-19150.

Ferrer-Mayorga, G., S. Alvarez-Diaz, N. Valle, J. De Las Rivas, M. Mendes, 


\section{Cystatins as regulators of cancer}

R. Barderas, F. Canals, O. Tapia, J.I. Casal, M. Lafarga, and A. Munoz. 2015. Cystatin D locates in the nucleus at sites of active transcription and modulates gene and protein expression. $J$ Biol Chem. 290:26533-26548.

Gocheva, V., and J.A. Joyce. 2007. Cysteine cathepsins and the cutting edge of cancer invasion. Cell Cycle. 6:60-64.

Gocheva, V., W. Zeng, D. Ke, D. Klimstra, T. Reinheckel, C. Peters, D. Hanahan, and J.A. Joyce. 2006. Distinct roles for cysteine cathepsin genes in multistage tumorigenesis. Genes Dev. 20:543-556.

Hunten, S., and H. Hermeking. 2015. p53 directly activates cystatin D/CST5 to mediate mesenchymal-epithelial transition: a possible link to tumor suppression by vitamin D3. Oncotarget. 6:15842-15856.

Jin, L., Y. Zhang, H. Li, L. Yao, D. Fu, X. Yao, L.X. Xu, X. Hu, and G. Hu. 2012. Differential secretome analysis reveals CST6 as a suppressor of breast cancer bone metastasis. Cell Res. 22:1356-1373.

Kashuba, E., J. Bailey, D. Allsup, and L. Cawkwell. 2013a. The kininkallikrein system: physiological roles, pathophysiology and its relationship to cancer biomarkers. Biomarkers. 18:279-296.

Kashuba, E., G.L. Eagle, J. Bailey, P. Evans, K.J. Welham, D. Allsup, and L. Cawkwell. 2013b. Proteomic analysis of B-cell receptor signaling in chronic lymphocytic leukaemia reveals a possible role for kininogen. J Proteomics. 91:478-485.

Klose, A., P. Zigrino, R. Dennhofer, C. Mauch, and N. Hunzelmann. 2006. Identification and discrimination of extracellularly active cathepsins B and $\mathrm{L}$ in high-invasive melanoma cells. Anal Biochem. 353:57-62.

Li, C., L. Chen, J. Wang, L. Zhang, P. Tang, S. Zhai, W. Guo, N. Yu, L. Zhao, M. Liu, and S. Yang. 2011. Expression and clinical significance of cathepsin B and stefin A in laryngeal cancer. Oncol Rep. 26:869-875.

Lin, Y.Y., Z.W. Chen, Z.P. Lin, L.B. Lin, X.M. Yang, L.Y. Xu, and Q. Xie. 2016. Tissue Levels of Stefin A and Stefin B in Hepatocellular Carcinoma. Anat Rec (Hoboken). 299:428-438.

McIntire, S.a.J.C. 2015. The effect of cystatin $\mathrm{C}$ construct clones on B16F10 in vitro cell behavior. Journal of Molecular Biochemistry. 5.

Nishiyama, K., A. Konishi, C. Nishio, K. Araki-Yoshida, H. Hatanaka, M. Kojima, Y. Ohmiya, M. Yamada, and H. Koshimizu. 2005. Expression of cystatin $\mathrm{C}$ prevents oxidative stress-induced death in PC12 cells. Brain Res Bull. 67:94-99.

Olson, O.C., and J.A. Joyce. 2015. Cysteine cathepsin proteases: regulators of cancer progression and therapeutic response. Nat Rev Cancer. 15:712729.

Pulukuri, S.M., B. Gorantla, J.A. Knost, and J.S. Rao. 2009. Frequent loss of cystatin E/M expression implicated 


\section{Cystatins as regulators of cancer}

in the progression of prostate cancer. Oncogene. 28:2829-2838.

Qiu, J., L. Ai, C. Ramachandran, B. Yao, S. Gopalakrishnan, C.R. Fields, A.L. Delmas, L.M. Dyer, S.J. Melnick, A.T. Yachnis, P.H. Schwartz, H.A. Fine, K.D. Brown, and K.D. Robertson. 2008. Invasion suppressor cystatin E/M (CST6): high-level cell type-specific expression in normal brain and epigenetic silencing in gliomas. $L a b$ Invest. 88:910-925.

Rivenbark, A.G., and W.B. Coleman. 2009. Epigenetic regulation of cystatins in cancer. Front Biosci (Landmark Ed). 14:453-462.

Shree, T., O.C. Olson, B.T. Elie, J.C. Kester, A.L. Garfall, K. Simpson, K.M. BellMcGuinn, E.C. Zabor, E. Brogi, and J.A. Joyce. 2011. Macrophages and cathepsin proteases blunt chemotherapeutic response in breast cancer. Genes Dev. 25:2465-2479.

Shridhar, R., J. Zhang, J. Song, B.A. Booth, C.G. Kevil, G. Sotiropoulou, B.F. Sloane, and D. Keppler. 2004. Cystatin M suppresses the malignant phenotype of human MDA-MB435S cells. Oncogene. 23:22062215.

Small, D.M., R.E. Burden, J. Jaworski, S.M. Hegarty, S. Spence, J.F. Burrows, C. McFarlane, A. Kissenpfennig, H.O. McCarthy, J.A. Johnston, B. Walker, and C.J. Scott. 2013. Cathepsin S from both tumor and tumorassociated cells promote cancer growth and neovascularization. Int $J$ Cancer. 133:2102-2112.
Sotiropoulou, G., A. Anisowicz, and R. Sager. 1997. Identification, cloning, and characterization of cystatin $\mathrm{M}$, a novel cysteine proteinase inhibitor, down-regulated in breast cancer. $J$ Biol Chem. 272:903-910.

Strojan, P., A. Anicin, B. Svetic, L. Smid, and J. Kos. 2011. Proteolytic profile of cysteine proteases and inhibitors determines tumor cell phenotype in squamous cell carcinoma of the head and neck. Int $J$ Biol Markers. 26:247-254.

Tang, N., Q. Xie, X. Wang, X. Li, Y. Chen, X. Lin, and J. Lin. 2011. Inhibition of invasion and metastasis of MHCC97H cells by expression of snake venom cystatin through reduction of proteinases activity and epithelial-mesenchymal transition. Arch Pharm Res. 34:781-789.

Turk, V., V. Stoka, and D. Turk. 2008. Cystatins: biochemical and structural properties, and medical relevance. Front Biosci. 13:5406-5420.

Wegiel, B., T. Jiborn, M. Abrahamson, L. Helczynski, L. Otterbein, J.L. Persson, and A. Bjartell. 2009. Cystatin $\mathrm{C}$ is downregulated in prostate cancer and modulates invasion of prostate cancer cells via MAPK/Erk and androgen receptor pathways. PLoS One. 4:e7953.

Weiss-Sadan, T., I. Gotsman, and G. Blum. 2017. Cysteine proteases in atherosclerosis. FEBS $J$.

Xie, Q., N. Tang, Y. Lin, X. Wang, X. Lin, and J. Lin. 2013a. Recombinant adenovirus snake venom cystatin inhibits the growth, invasion, and 
Medical Research Archives. Volume 5, issue 7. July 2017.

\section{Cystatins as regulators of cancer}

metastasis of B16F10 cells in vitro and in vivo. Melanoma Res. 23:444451.

Xie, Q., N. Tang, R. Wan, Y. Qi, X. Lin, and J. Lin. 2013b. Recombinant snake venom cystatin inhibits tumor angiogenesis in vitro and in vivo associated with downregulation of VEGF-A165, Flt-1 and bFGF. Anticancer Agents Med Chem. 13:663-671.

Yan, Y., K. Zhou, L. Wang, Y. Zhou, X. Chen, and Q. Fan. 2015. Expression of cystatin $\mathrm{C}$ and its effect on EC9706 cells in esophageal carcinoma. Int J Clin Exp Pathol. 8:10102-10111.

Zajc, I., I. Hreljac, and T. Lah. 2006. Cathepsin L affects apoptosis of glioblastoma cells: a potential implication in the design of cancer therapeutics. Anticancer Res. 26:3357-3364.

Zhang, J., R. Shridhar, Q. Dai, J. Song, S.C. Barlow, L. Yin, B.F. Sloane, F.R. Miller, C. Meschonat, B.D. Li, F. Abreo, and D. Keppler. 2004. Cystatin m: a novel candidate tumor suppressor gene for breast cancer. Cancer Res. 64:6957-6964. 